\title{
Incidencia y tipo de efectos adversos durante el procedimiento de hemodiálisis
}

\author{
Estela María Matarán Robles, Rafael Aguilar García, Mercedes Muñoz Becerra
}

Enfermeros/as. Unidad de hemodiálisis, Hospital Virgen de la Nieves de Granada

\section{Resumen}

\section{Introducción}

La seguridad del paciente constituye hoy día una prioridad para las principales organizaciones de salud como la Organización Mundial de la Salud, organismos internacionales como la Unión Europea, el Consejo de Europa, autoridades sanitarias, sociedades profesionales y organizaciones de pacientes.

\section{Objetivo}

Describir las incidencias y tipos de efectos adversos derivados de la hemodiálisis en una unidad de nefrología.

\section{Metodología}

Diseño: Estudio transversal en una unidad de hemodiálisis de 28 camas.

Variables: como variable dependiente se consideró la presencia de efectos adversos y tipología, para ello se utilizó la Clasificación Internacional para la Seguridad del Paciente.

Análisis estadísticos: se realizó una descripción de la muestra, para ello se utilizaron tanto los gráficos (histogramas, diagramas de barras y dispersión) como los porcentajes, medias, desviaciones típicas, medianas, etc.

\section{Resultados}

Durante el periodo a estudio se practicaron un total de 4.797 procedimientos de hemodiálisis, en un to-

\section{Correspondencia: \\ Estela María Matarán Robles \\ c/ Maestro Montero $n^{\circ} 21$ portal 1B-piso $3^{\circ} \mathrm{B}$ \\ 18004, Granada \\ E-mail: estely8@telefonica.net}

tal de 681 sesiones produjeron efectos adversos, lo que supone una tasa de incidencia de 141,96 cada 1.000 diálisis.

En cuanto a la gravedad el $97,5 \%$ de los efectos adversos fueron clasificados como leves, frente al $1,8 \%$ y $0,7 \%$ que fueron considerados como moderados y graves respectivamente.

\section{Conclusiones}

Nuestra tasa de incidencia y gravedad de los efectos adversos es similar a la de otros centros. Este estudio nos permite identificar los problemas de seguridad en nuestra Unidad y en una segunda fase realizar un protocolo conjunto, que nos permita disminuir la tasa de incidencia actualmente existente. La hipotensión fue el efecto adverso más frecuente.

\section{PALABRAS CLAVE:}

- EFECTO ADVERSO

- HEMODIÁLISIS

\section{Incidence and type of adverse effects during the haemodialysis procedure}

\section{Abstract}

\section{Introduction}

Nowadays patient safety is a priority for the main health organizations such as the World Health Organization, international bodies such as the European Union, the Council of Europe, healthcare authorities, professional associations and patient organizations. 


\section{Objective}

To describe the incidence and types of adverse effects deriving from haemodialysis in a nephrology unit.

\section{Methodology}

Design: Transversal study in 28-bed haemodialysis unit.

Variables: as a dependent variable, the present of adverse effects and typology was considered, using the International Classification for Patient Safety. Statistical analyses: a description of the sample was carried out, using graphs (histograms, bar diagrams, and dispersion diagrams) and also percentages, means, standard deviations, medians, etc.

\section{Results}

During the period studied a total of 4797 haemodialysis procedures were carried out, with adverse effects arising in a total of 681 sessions, which represents an incidence rate of 141.96 per 1000 dialyses.

As far as severity is concerned, $97.5 \%$ of the adverse effects were classified as mild, compared to $1.8 \%$ and $0.7 \%$ which were considered moderate and serious, respectively.

\section{Conclusions}

Our incidence rate and severity of adverse effects is similar to other centres. This study lets us identify safety problems in our Unit and in a second phase establish a joint protocol which will allow us to reduce the current incident rate. Hypotension was the most common adverse effect.

\section{KEY WORDS:}

- ADVERSE EFFECT

- HAEMODIALYSIS

\section{Introducción}

La seguridad del paciente constituye hoy en día una prioridad para las principales organizaciones de Salud como la Organización Mundial de la Salud, organismos internacionales como la Unión Europea el Consejo de Europa, autoridades sanitarias, sociedades profesionales y organizaciones de pacientes.

Tal y como se concibe desde el Ministerio de Sanidad(1) la seguridad del paciente, componente de la calidad asistencial , ha adquirido gran relevancia en los últimos años tanto para los pacientes como para sus familias, que desean sentirse seguros y confiados en los cuidados sanitarios recibidos, como para los gestores y profesionales que deseen ofrecer una asistencia sanitaria segura y efectiva y eficiente.

Los efectos no deseados secundarios en la atención sanitaria representan una causa de elevada mortalidad en todos los sistemas sanitarios desarrollados. La razón fundamental es la creciente complejidad del manejo de los pacientes, en el que interactúan factores organizativos, factores personales de los profesionales y factores relacionados con la enfermedad. Los daños que puedan ocasionar a los pacientes en el ámbito sanitario y el coste que le suponen a los sistemas sanitarios son de tal relevancia que las principales organizaciones de salud como la Organización Mundial de la Salud, la Organización Pan Americana de la salud, el Comité de Sanidad del Consejo de Europa así como diversas agencias y organismos internacionales han desarrollado estrategias en los últimos años para proponer planes, acciones y medidas legislativas que permitan controlar los efectos adversos evitables en la práctica clínica.

La Junta de Andalucía, a la cual pertenece nuestro Centro en el ámbito autonómico, a través de su observatorio para la seguridad del paciente participa actualmente en la acción conjunta "Red de la Unión Europea para la seguridad del paciente y la calidad asistencial".

Las actividades que se realizan en una sala de hemodiálisis son variables y complejas. Los riesgos de un paciente que va a ser sometido a una sesión de hemodiálisis son muy diversos y de diferente importancia. Inicialmente debemos de conocer cuales son los mismos para poder tratar de evitarlos.

Existen muy pocos estudios que analicen los efectos adversos, y recojan prácticas preventivas dirigidas a mejorar la seguridad del paciente durante su asistencia en una Unidad de hemodiálisis.

Nuestro objetivo es describir la incidencia y tipo de efecto adverso derivados de la hemodiálisis en una Unidad de Nefrología de un Hospital de tercer nivel. 


\section{Material y métodos}

El ámbito del estudio se realiza en la Unidad de Gestión Clínica de Nefrología: Unidad de Hemodiálisis, del Hospital de tercer nivel Virgen de las Nieves de Granada, con un total de veintiocho camas. Se realizó entre octubre del 2011 y octubre del 2012. El procedimiento seguido fue la recogida de y cumplimentación de la base de datos que figura en la TABLA N ${ }^{\circ} 1$, por parte del personal de enfermería de dicha Unidad de Hemodiálisis. Dicha hoja de cumplimentación requirió de una elaboración previa con las bases de datos: medline, embase, CINAHL, Cochrane, y otros recursos en la categoría de medicina basada en la evidencia: uptodate, y publicaciones de enfermería. Se elaboró un listado de efectos adversos y causas, el cual fue presentado en nuestra Unidad de gestión para dar a conocer dicho plan y la aprobación del mismo. Se utilizaron las siguientes definiciones:

- Incidente: Evento o circunstancia que resultó o podría haber resultado en un daño innecesario a un paciente.

- Incidente sin daño: Incidente que no causa daño.

- Efecto adverso: Incidente que produce daño no intencionado a un paciente.

En función de la gravedad del mismo se estratificaron en tres grupos.

1. LEVE: Aquel que ocasiona lesión o complicación sin prolongación de la estancia hospitalaria.

2. MODERADO: Aquel que ocasiona prolongación de la estancia hospitalaria al menos de 1 día de duración.

3. GRAVE: Aquel que ocasiona exitus o incapacidad residual al alta hospitalaria o que requirió intervención quirúrgica.

Diseño del estudio: Estudio transversal en una Unidad de hemodiálisis de 28 camas.

Análisis estadístico: Se realizó con el programa SPSS vs 17.0 para Windows. Inicialmente se procedió a introducir todos los efectos adversos en el periodo temporal ya descrito, se codificaron las variables y se realizo un estudio descriptivo, para ello se utilizaron tanto los gráficos (histogramas, diagramas de barras y dispersión) como los porcentajes, medias, desviaciones típicas, y medianas.

\section{Resultados}

Durante el periodo a estudio se practicaron un total de 4.797 procedimientos de hemodiálisis, en un total de 681 sesiones (procedimientos) se produjeron efectos adversos (EA), lo que supone una tasa de incidencia de 141,96 EA cada 1.000 diálisis. Estos 681 se produjeron en un total de 94 pacientes, no se encontraron diferencias temporales a lo largo del estudio. La edad media de los pacientes en hemodiálisis fue de 65,34 años (DT 12,36).

En el total de 681 sesiones de hemodiálisis, de ellas en el $87,2 \%$ de las sesiones se produjo un único $E A$, $10,1 \%$ dos EA y tres EA en el $2,6 \%$.

En cuanto a la gravedad el $97,5 \%$ de los eventos fueron clasificados como leves, frente al $1,8 \%$ y $0,7 \%$ que fueron considerados como moderados y graves respectivamente.

El efecto adverso más frecuente fue la hipotensión la cual se produjo en el $55,4 \%$ de los procedimientos, seguido de inadecuado tratamiento dialítico en el $20,1 \%$ de los procedimientos, coagulación en el $7.5 \%$ de los mismos, e infección en el acceso vascular en el $2.3 \%$ de los procedimientos.

El resto de los efectos adversos un porcentaje se encuentra entre el $0,1 \%$ y el $1,3 \%$. Si es destacable solo 2 caídas en un total de 681 procedimientos.

\section{Discusión}

El Ministerio de Sanidad, servicios Sociales e Igualdad, en su responsabilidad de mejorar la calidad del sistema sanitario en su conjunto, ha situado la seguridad del paciente en el centro de las políticas sanitarias como uno de los elementos clave de la mejora de la calidad, quedando así reflejado en la estrategia $n^{\circ} 8$ del plan de calidad para el sistema nacional de salud, que se viene desarrollando desde el 2005 en coordinación con las comunidades autónomas ${ }^{(1,2)}$. La nueva Estrategia para la Seguridad del Paciente en la Comunidad autónoma Andaluza (2011-2014) nace con el claro reto de consolidar la cultura de seguridad en el seno del Sistema Sanitario Público Andaluz, a fin de preservar el derecho básico de los pacientes a recibir una atención sanitaria segura y de calidad(3). 
Los cuidados enfermeros durante el tratamiento de hemodiálisis son muy importantes para que la asistencia que reciba sea de calidad. La combinación de un procedimiento que exige interacción humana y alta tecnología en un grupo de pacientes con una patología crónica que suele llevar aparejada otras comorbilidades, hace que dicho procedimiento este expuesto a numerosos riesgos, que en su mayoría son evitables. De todos modos todavía se sigue hablando de complicaciones más 0 menos frecuentes durante la sesión de hemodiálisis en vez de eventos adversos ${ }^{(4)}$ en los que sin duda los profesionales de enfermería juegan un papel fundamental dado que son los primeros en detectar e intervenir en tales "complicaciones", al estar en continuo contacto con los pacientes.

Nuestro catalogo de posibles efectos adversos que figura en la tabla $n^{\circ} l$ es muy similar al ya publicado en 2010 por el banco de prácticas innovadoras (saludinnova.com) de la consejería de salud de la Junta de Andalucía, y de hecho nos sirvió de base para la realización del nuestro ${ }^{(5)}$.

El trabajo de Holley del $2006^{(6)}$ realizado en unidades de hemodiálisis de USA afirma que no existen estudios que describan el tipo y frecuencia de efectos adversos en Unidades de Hemodiálisis. En el mismo la hipotensión que figura como "complicación frecuente" en una Unidad de hemodiálisis en las publicaciones ${ }^{(3,6)}$ ni siquiera aparece en este estudio, siendo en el nuestro el efecto adverso más frecuente $(55,4 \%)$ y evitable si se tomaran las medidas oportunas previas al procedimiento dialítico: peso correcto, no ultrafiltración excesiva, etc), al igual que no reporta datos sobre inadecuado tratamiento dialítico. El resto de los datos son difícilmente comparables, los problemas del circuito de diálisis que son en su estudio los más frecuentes no sabemos exactamente que incluyen, por lo tanto es difícil comparar la tasa de incidencia en ambos estudios. Si podemos comparar el porcentaje de caídas que en su estudio está en el $0.013 \%$ y en el nuestro en el $0.3 \%$, el de infiltración subcutánea de la vía venosa 0,04\% frente a $1,3 \%$ en nuestro caso, y un $0,05 \%$ errores en la medicación que en nuestro caso sube al $0,6 \%$, aunque con exactitud los registros no se pueden comparar de una forma $100 \%$ fiable. Lo que es difícil de creer en este estudio es que de 64.541 procedimientos de diálisis en ningún caso el paciente requirió hospitalización como consecuencia del mismo, en nuestro caso el $0,7 \%$ de 4797 procedimientos. Este estudio nos permite identificar los problemas de seguridad de nuestra Unidad y en una segunda fase realizar un protocolo conjunto medico enfermera, que nos permita disminuir la tasa de incidencia actualmente existente. La existencia de un registro de efectos adversos permitirá tomar medidas preventivas y mejorar la asistencia sanitaria a estos pacientes.

Recibido: 8 Enero 2013

Revisado: 14 Enero 2013

Modificado: 18 Febrero 2013

Aceptado: 20 Febrero 2013

\section{Bibliografía}

1. Seguridad del paciente. Ministerio de Sanidad, Servicios Sociales e Igualdad.

Acceso febrero 2013. Disponible en http://www. seguridaddelpaciente.es.

2. Aranaz JM,Aibar C, Vitaller J, Ruiz P.Estudio nacional sobre efectos adversos ligados a la hospitalización.Eneas. Madrid.Ministerio de sanidad y Consumo.2006.

3. Observatorio para la seguridad del paciente.Junta de Andalucía.Agencia de calidad Sanitaria.

Acceso febrero 2013.Disponible en http://juntadeandalucia.es/agenciadecalidadsanitariadeandalucia/observatorioseguridaddelpaciente.

4. Rojo Tordable M, Sánchez Cano MS, Cepa García H.Atención de enfermería durante la sesión de hemodiálisis.En Alonso Nantes Rr; Pelayo Alonso R.Manual de Enfermería Nefrológica.Barcelona: Ediciones Pulso;2012:165-191.

5. Plan de Seguridad del paciente en hemodiálisis. María Antonia Álvarez de Lara Sánchez.Saludinnova.com.Banco de practicas innovadoras.fecha de publicación: 21/04/2010. Disponible en: http:// www.saludinnova.com/practices.

6. Holley JL. A descriptive report of error and adverse events in chronic hemodialysis Units. Nephrol News Issues. 2006; 20(12):57-63.

7. Aranaz JM,Aibar C, Vitaller J, Ruiz P.Estudio nacional sobre efectos adversos ligados a la hospitalización.Eneas. Madrid.Ministerio de sanidad y Consumo.2006. 


\section{Tabla $n^{0} 1$. NOTIFICACIÓN DE EFECTOS ADVERSOS EN HEMODIÁLISIS}

\section{SERVICIO DE NEFROLOGÍA HOSPITAL VIRGEN DE LAS NIEVES DE GRANADA}

Definición EFECTO ADVERS0: Incidente que produce daño no intencionado a un paciente.

Definición de INCIDENTE: Evento o circunstancia que resultó o podría haber resultado en un daño innecesario a un paciente.

HIPOTENSION/UF excesiva, ingesta durante diálisis, Error
al pesar, peso seco inadecuado
EMBOLIA GASEOSA/Dejar abierto catéter arterial, avería
sistema detección aire etc
HEMÓLISIS
IAM, ACV, O TEP DURANTE O DESPUES.EXITUS
COAGULACIÓN DEL SISTEMA
INFECCIÓN DEL ACCESO VASCULAR
SEGURIDAD EN EL USO DE MEDICAMENTOS
TRANSMISION DE ENFERMEDADES VIRALES
REACCIÓN ADVERSA MEDICAMENTOSA

Definición Incidente sin daño: Incidente que no causa daño.

Información sobre el evento adverso:

\section{Etiqueta del paciente}

Fecha de notificación:

SALIDAS DE AGUJAS DURANTE LA DIÁLISIS

INADECUADO TRATAMIENTO DIALÍTICO (inversión

líneas, tiempo diálisis erróneo)

SANGRADO EXCESIVO POR PUNTOS DE PUNCIÓN

INFILTRACIÓN SUBCUTANEA

CAIDAS DURANTE LA DIÁLISIS

CALAMBRES

ERROR EN LA PETICIÓN DE ANALÍTICA

OTROS

Fecha del suceso:

1. LEVE: Aquel que ocasiona lesión o complicación sin prolongación de la estancia hospitalaria.

2. MODERADO: Aquel que ocasiona prolongación de la estancia hospitalaria al menos de 1 día de duración.

3. GRAVE: Aquel que ocasiona exitus o incapacidad residual al alta hospitalaria o que requirió intervención quirúrgica.

\section{GRAVEDAD DEL MISMO (TACHAR LO QUE PROCEDA)}

LEVE MODERADO GRAVE

\section{MEDIDAS ADOPTADAS ANTE EL EFECTO ADVERSO}

\section{DATOS DEL PROFESIONAL QUE LO NOTIFICA (TACHAR LO QUE PROCEDA)}

Personal Médico

Personal Auxiliar
Personal de Enfermería

Otros: 